\title{
Correlation between degree of crystallinity, morphology and mechanical properties of PPS/carbon fiber laminates
}

\author{
Natassia Lona Batista ${ }^{a, b *}$, Philippe Olivier ${ }^{b}$, Gérard Bernhart ${ }^{b}$, Mirabel Cerqueira Rezende ,
}

\author{
Edson Cocchieri Botelho ${ }^{a}$
}

\author{
${ }^{a}$ Department of Materials and Technology, Universidade Estadual Paulista Júlio de Mesquita Filho - UNESP, \\ Av. Ariberto Pereira da Cunha, 333, Guaratinguetá, SP, Brazil \\ ${ }^{b}$ Institut Clément Ader, 3 rue Caroline Aigle, 31400, Toulouse, France \\ 'Institute of Science and Technology, Universidade Federal de São Paulo - UNIFESP, \\ Rua Talim, 330, São José dos Campos, SP, Brazil
}

Received: August 9, 2015; Revised: November 10, 2015; Accepted: November 26, 2015

\begin{abstract}
The crystallization degree in semi-crystalline thermoplastics plays an important role in determining the final properties of structural composite material (e.g. toughness, stiffness and solvent resistance). The main purpose of this work is to study different induced degrees of crystallinity in carbon fiber (CF) reinforced polyphenylene sulfide (PPS) composites, by using three different cooling rates during hot compression molding processing ( $51 \%, 58 \%$ and $62 \%$ of crystallinity). In this study, the morphology, thermal and mechanical properties of the produced laminates were investigated and compared. The results showed an increase in the storage modulus (9.8\%), Young's modulus $(9.2 \%)$ and ILSS $(14.2 \%)$ for the lower cooling rates. Evidences of fiber/interface improvement and crystallites nucleation on the fiber reinforcement surface were also identified.
\end{abstract}

Keywords: CF/PPS, Thermoplastic composites, Crystallinity.

\section{Introduction}

PPS is a semi-crystalline thermoplastic with a symmetrical rigid backbone chain consisting of recurring para-substituted rings and sulphur atoms ${ }^{1,2}$. This high-performance polymer exhibits a glass transition temperature $\left(\mathrm{T}_{\mathrm{g}}\right)$ of about $90^{\circ} \mathrm{C}$ and a melting temperature $\left(\mathrm{T}_{\mathrm{m}}\right)$ around $285^{\circ} \mathrm{C}^{3}$. It presents outstanding physical and chemical properties such as high-temperature stability, inherent flame resistance, good chemical resistance, anti-aging, high hardness and rigidity and excellent friction properties ${ }^{2,4}$. Due mainly to its low melt viscosity ( 200 Pa.s), the PPS thermoplastic polymer can be molded with high filler or reinforcement contents 3,5. Most PPS products are reinforced with carbon or glass fibers for high-performance applications, including electronic, automotive and aerospace ones ${ }^{6}$. Carbon fiber reinforced PPS (CF/PPS) is mainly used in aircraft structural applications, such as in J-Nose wing substructures of the Airbus A340-500/600 7 .

PPS is also well known for being able to attain a high level of crystallinity, with the commercial grades achieving crystallinities of $60 \%{ }^{8}$. Crystallinity degree in high performance thermoplastics is important, because it has a strong influence on chemical and mechanical properties: the crystalline phase tends to increase stiffness and tensile strength, while the amorphous phase is more effective in absorbing impact energy ${ }^{9}$. When a semi-crystalline thermoplastic is cooled from the melt state, it normally forms spherical structures so called "spherulites". Spherulites have been described as approximately radially symmetric semi-crystalline structures.

*e-mail: natassia_lb@hotmail.com
Considered from a central point, each spherulite consists of a radial assembly of thin crystalline lamellae which are separated by amorphous layers. The crystalline domain is formed by regularly ordered molecular chains, while the amorphous phase consists of an assembly of disordered macromolecules, which are morphologically constrained by the neighboring crystalline lamellae ${ }^{10}$. The dimension range of spherulites varies from micrometers to millimeters, depending on the structure of the polymer chain and the crystallization conditions, such as cooling rate, crystallization temperature, and the content of the nucleating agent ${ }^{11}$. When these structures are viewed between cross polarizers, they exhibit a characteristic "Maltese cross" extinction pattern ${ }^{12}$.

For semi-crystalline polymer composites, the fillers can have the potential to induce or alter polymer crystallization, acting as nucleating agents. Thus, an increase in crystallization rate and crystallization temperature as well as a decrease in crystal size is generally observed ${ }^{13}$. The crystalline formation at the interphase region depends on a number of factors, such as matrix morphology, substrate surface condition, as well as the presence of reactive functionalities ${ }^{14}$.

Some fibers may induce crystallization along the polymer/fiber interface due to the high density of active nuclei on its surface, which hinder the full extension of spherulites and force the crystal growth in the perpendicular direction ${ }^{13}$. This particular behavior can results in a columnar crystalline layer, known as transcrystallinity (TC ${ }^{15}$. Since it was first reported, TC has attracted tremendous attention, because it may be an effective and economical method to 
improve the interfacial adhesion between polymer matrix and fiber. To date, TC has been reported to occur at the interface between several semi-crystalline polymers, such as PPS and PEEK ${ }^{13}$.

Several different routes have been developed for manufacturing continuous fiber-reinforced thermoplastic composites. These composite materials can be obtained by using pre-impregnation techniques, such as hot-melt impregnation, fluid/bed impregnation and solvent-based impregnation; or post-shaping impregnation techniques, such as powder suspension impregnation, film stacking and commingling of thermoplastic fibers with reinforcing fibers ${ }^{16-18}$. Hot compression molding process is a widely used technique to produce thermoplastic-matrix-composite laminates from these raw materials. The consolidation is achieved by placing the plies with proper sequence into the die cavity, heating it up and keeping the material under pressure at temperatures higher than the thermoplastic melting/softening point until the impregnation process between the plies is completed. Finally, the composite is cooled down to the room temperature ${ }^{7}$. For non-flat dies, the post-shaping impregnation techniques are more suitable due to the drapeability of the fabrics obtained by this process.

The aim of this work is to bring a better understand of the crystallization process, under three different cooling rates, of PPS polymer on the carbon fiber (CF) surfaces and evaluate the mechanical properties of CF/PPS composites. The influence of crystallinity on the laminates was evaluated by thermal and mechanical characterization applying differential scanning calorimetry (DSC), dynamic mechanical analysis (DMA), interlaminar shear strength (ILSS) and acoustic vibration damping.

\section{Material and methods}

\subsection{Material}

Thermoplastic laminates were processed by PPS films (supplied by Curbell Plastics) and plain weave carbon fiber fabric (provided by Hexcel) film stacking technique. The final laminates were produced with $2.5 \mathrm{~mm}$ of thickness, attending the reinforcement/matrix volume content of $60 / 40(\mathrm{v} / \mathrm{v})$. In order to obtain this result, 15 layers of reinforcement and polymer films were required.

\subsection{Polarized light optical microscopy}

The crystallization process of PPS around a microfilament of carbon fiber was observed on thin films using a polarized light optical microscope (Laica-DMLS) with automatic heating and cooling stages (Linkam THMS600). The samples, placed between two glass covers, were heated up from room temperature to $315^{\circ} \mathrm{C}$ at $10^{\circ} \mathrm{C} \cdot \mathrm{min}^{-1}$, kept in this temperature during $10 \mathrm{~min}$ and cooled down in the following different rates: $0.5,1,5,10$ and $20^{\circ} \mathrm{C} \cdot \mathrm{min}^{-1}$.

\subsection{Rheology}

Rheological tests were performed on PPS film samples using a parallel plate rheometer with controlled deformation (Thermo Scientific HAAKE MARS CTC), in order to establish the maximum period of time that the material could be kept inside the die before cooling. The linear rheological response was obtained from one hour long analysis by heating the polymer $\left(10{ }^{\circ} \mathrm{C} \cdot \mathrm{min}^{-1}\right)$ until $315^{\circ} \mathrm{C}$ with frequency of $1 \mathrm{~Hz}$ and $1 \%$ of deformation.

\subsection{Hot compression molding process}

The composite laminate consolidation process was carried out in a steel die with dimensions of $300 \mathrm{~mm}$ x $300 \mathrm{~mm}$. Firstly, the material was heated from room temperature $\left(\sim 25^{\circ} \mathrm{C}\right)$ up to $315^{\circ} \mathrm{C}$ at approximately $10^{\circ} \mathrm{C} \cdot \mathrm{min}^{-1}$, held in this temperature for $30 \mathrm{~min}$ under $1.2 \mathrm{MPa}$ of pressure and then cooled down to room temperature. These parameters were stipulated according to the literature found on this subject ${ }^{1,3-7,19,20}$ and preliminary performed tests. In this work, three different cooling rates were applied in order to vary the final degree of crystallization, a fast rate of about $10^{\circ} \mathrm{C} \cdot \mathrm{min}^{-1}$, a slow rate of approximately $1^{\circ} \mathrm{C} \cdot \mathrm{min}^{-1}$ and an air cooling, obtained by leaving the press, die and laminate cool naturally in air. Figure 1 shows the processing cycle used to obtain the PPS/carbon fiber laminates.

\subsection{Differential Scanning Calorimetry (DSC)}

The crystallization of the processed composites was investigated by DSC using a Seiko Exstar 6000 - DSC 6220 differential scanning calorimeter operating under nitrogen flow. Samples (10mg) were cut from CF/PPS laminates and sealed in aluminum pans. A heating rate of $10{ }^{\circ} \mathrm{C} \cdot \mathrm{min}^{-1}$ was used to heated the samples from room temperature $\left(25^{\circ} \mathrm{C}\right)$ up to $315^{\circ} \mathrm{C}$.

The melting enthalpy of each sample was obtained from the area under the melting peaks. The levels of crystallinity were calculated by dividing the melting enthalpy of the samples by the melting enthalpy of PPS $100 \%$ crystalline is $80 \mathrm{~J}^{-g^{-1} 3}$. However, since the specimens contain polymer and carbon fiber, the reinforcement mass had to be subtracted from the total. Therefore, the levels of crystallinity of PPS composites were determined according to Equation 1:

$$
X_{c}(\%)=\frac{\Delta H_{c}}{\Delta H^{\circ}{ }_{m}(1-x)} \times 100 \%
$$

where: $X_{C}$ is the degree of crystallinity, $\Delta \mathrm{H}_{C}$ is obtained by the melting peak area, $\Delta \mathrm{H}^{\circ}{ }_{M}$ is the melting enthalpy of PPS $100 \%$ crystalline, and $\mathrm{x}$ is fiber weight fraction.

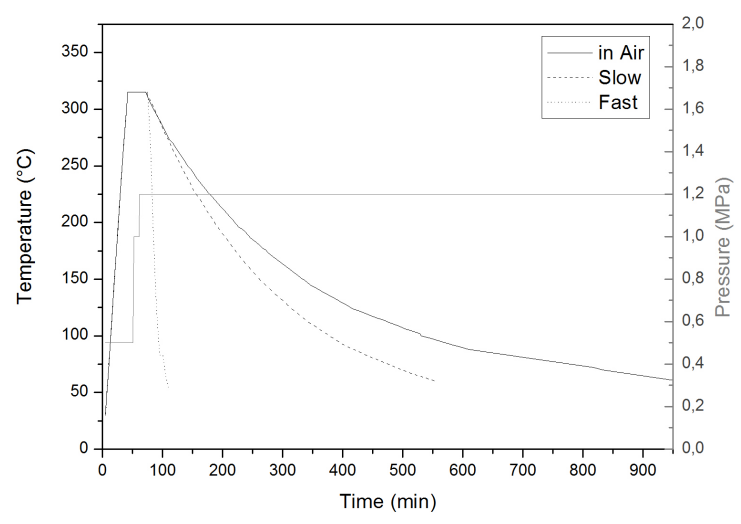

Figure 1. Consolidation cycle used for laminate manufacturing. 


\subsection{Dynamic Mechanical Analysis (DMA)}

The dynamic mechanical performance of the composite samples was analyzed using a Seiko SII Exstar 6000 dynamic mechanical analyzer. Experiments were performed in dual cantilever bending mode at frequency of $1 \mathrm{~Hz}$. A dynamic force of $2 \mathrm{~N}$ was used at fixed frequency and amplitude of $10 \mu \mathrm{m}$. The results were recorded in the temperature range of 30 to $200^{\circ} \mathrm{C}$, at a heating rate of $3{ }^{\circ} \mathrm{C} \cdot \mathrm{min}^{-1}$. The dimensions of the specimens analyzed were approximately of $50 \mathrm{~mm}$ x $14 \mathrm{~mm}$ x $2.5 \mathrm{~mm}$.

\subsection{Interlaminar Shear Strength (ILSS)}

Interlaminar shear strength measurements were carried out using a Shimadzu autograph AG-X series precision universal machine at room temperature, using a constant cross-speed of $1 \mathrm{~mm} \cdot \mathrm{min}^{-1}$ and a load cell of $5 \mathrm{kN}$. The tests were performed according to ASTM D2344 standard, using at least five specimens with dimensions of $15 \mathrm{~mm} \times 5.5 \mathrm{~mm} \times 2.5 \mathrm{~mm}$.

\subsection{Determination of Young's modulus}

The impulse excitation of vibration was used to determine Young's modulus from the natural frequency, using a rectangular specimen under flexure mode. At the technique applied, the specimen is lightly stroked, the acoustic response is captured and this is processed by software, which calculates the Young's modulus. The impulse excitation technique was applied using Sonelastic ${ }^{\circledR}$ equipment, developed by ATCP - Physical Engineering, at Brazil. This non-destructive test was performed according to ASTM 1876 standard, using specimens with dimension of $50 \mathrm{~mm} \times 14 \mathrm{~mm} \times 2.5 \mathrm{~mm}$.

\section{Results and discussion}

\subsection{Crystallization morphology}

It is well known that the crystallization process plays a crucial role on the properties of semi-crystalline polymers ${ }^{12-14}$ and that the presence of carbon fibers as reinforcement may also influence this process ${ }^{15-17}$. However, the images obtained by polarized light optical microscopy in this work do not show any evidence of nucleation under the cooling rates performed, when analyzed in the range of 0.5 to $20^{\circ} \mathrm{C} / \mathrm{min}$. The crystal growing seemed to take place in a homogenous manner, even for the slowest cooling, as it can be observed in Figure 2.

Although the PPS crystallites formation was highly homogeneous, a different behavior was observed for the cooling performed at $5{ }^{\circ} \mathrm{C} / \mathrm{min}$. Due to the formation of a bubble on the surface of a carbon fiber microfilament, the very low thickness of the created polymer film allowed the visualization of crystallites nucleation on the microfilament surface, as shown in Figure 3. As mentioned, it is believed that the observation of nucleation was only possible owing to the thickness of the film, since there were no crystallites overlapping. Moreover, the crystal growing continues to develop as before, in a very homogenous manner.

In addition to nucleation, a transcrystalline layer can also be seen on the carbon fiber surface (Figure 4(a)). Such phenomenon was not visualized on the bubble boundary far from the fiber, as showed in Figure 4(b), which excludes
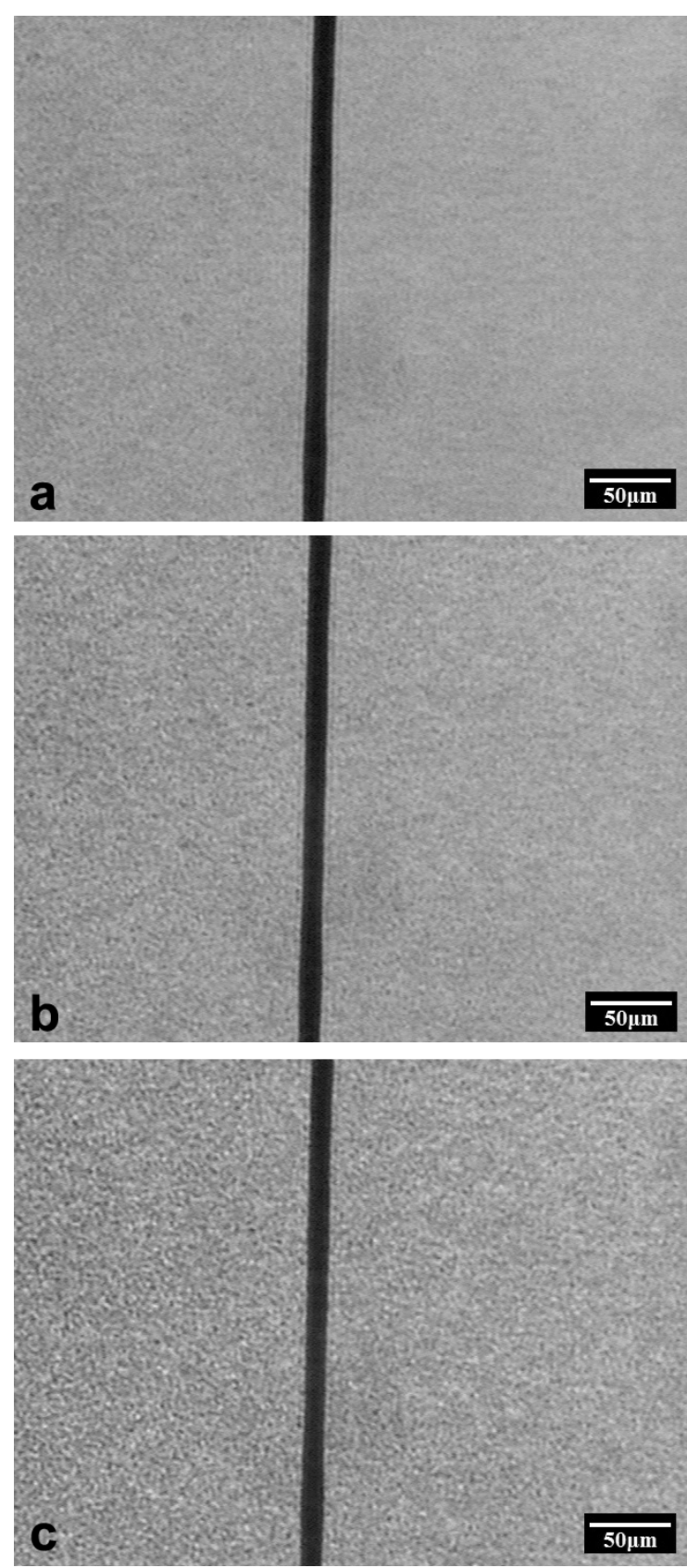

Figure 2. Polarized light optical micrographs of melted PPS cooling at $0.5^{\circ} \mathrm{C} / \mathrm{min}$ in the presence of a carbon fiber microfilament: (a) $277^{\circ} \mathrm{C}$; (b) $276^{\circ} \mathrm{C}$ and (c) $275^{\circ} \mathrm{C}$.

the possibility that the layer formed was a result of the bubble presence.

\subsection{Rheological investigation}

The rheological analysis performed on the PPS films (Figure 5) revels that before $30 \mathrm{~min}$, at a constant temperature of $315^{\circ} \mathrm{C}$, no significant increase on the viscosity is observed. This behavior not only facilitates the polymer percolation during the consolidation process, but also indicates that thermal degradation is not likely to occur in this period of 

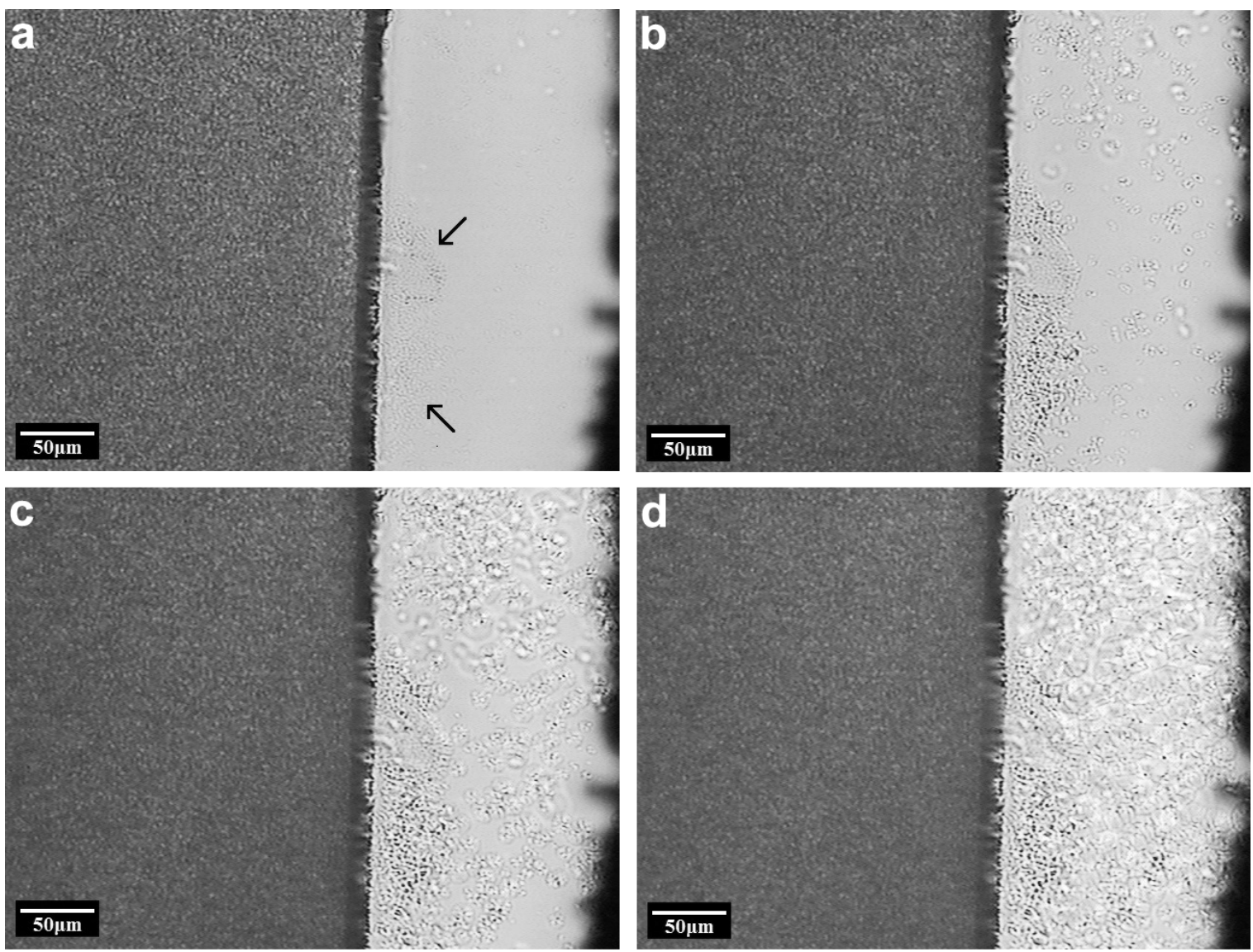

Figure 3. Polarized light optical micrographs of melted PPS cooling at $5{ }^{\circ} \mathrm{C} / \mathrm{min}$ in the presence of a carbon fiber microfilament: (a) $250{ }^{\circ} \mathrm{C}$; (b) $235^{\circ} \mathrm{C}$; (c) $230{ }^{\circ} \mathrm{C}$ and (d) $225^{\circ} \mathrm{C}$.
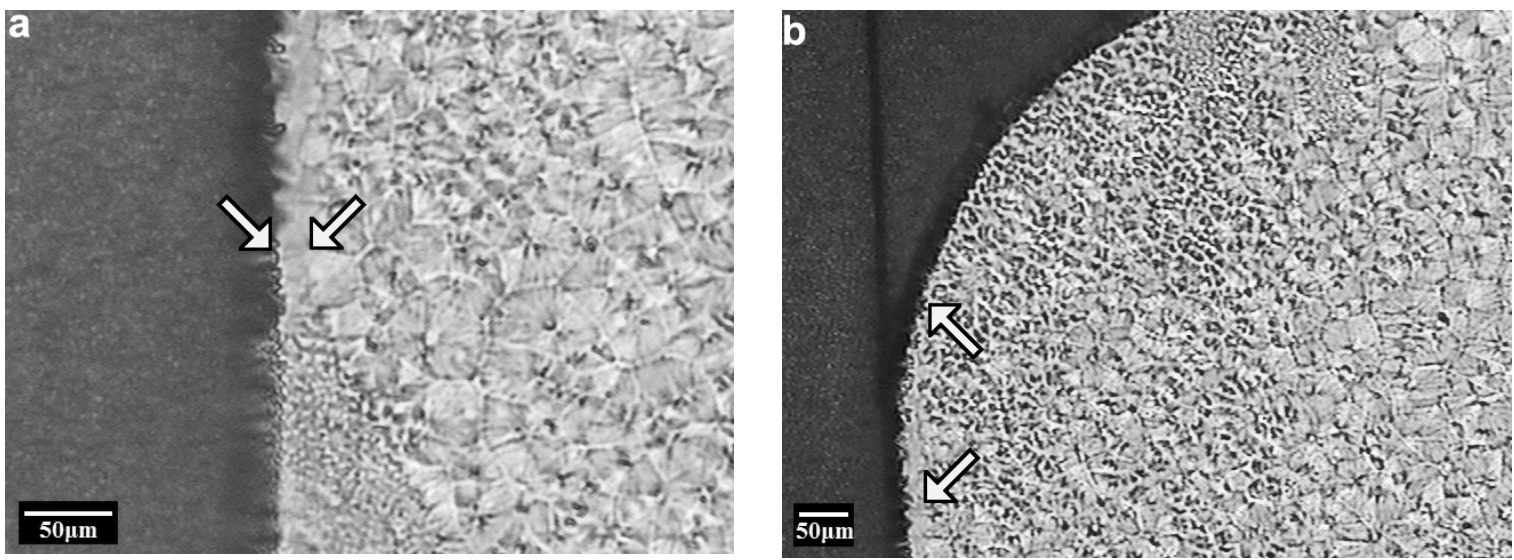

Figure 4. Polarized light optical micrographs of crystallized PPS cooled at $5{ }^{\circ} \mathrm{C} / \mathrm{min}$ in the presence of a carbon fiber microfilament: (a) transcrystallinity formation; (b) superior boundary limit of the formed bubble.

time. Therefore, the crystallization process would not be affected by thermal degradation.

\subsection{Melting behavior and crystalline content}

The DSC melting curves of the different manufactured laminates, concerning the cooling rate, are displayed in Figure 6. The melting peak area of the DSC curves increases with the decrease of the cooling rate due to the higher crystalline content into the PPS matrix. This behavior can be better observed in Table 1, where the melting enthalpy (or melting peak area) is used to obtain the crystallinity percentage of PPS in the PPS/carbon fiber composite.

In addition to the alteration in the area, two other differences in the peak are observed. Firstly, it is observed that 
the melting temperature increases from fast to in air cooling, which indicates that the solid polymer matrix resulting from the latter cooling rate has better ordered crystallites resulted from the longer cooling time. Therefore, longer cooling time favors the alignment and folding of the polymer chains forming regions more orderly. Secondly, the curve shape for the fast cooled samples also differs from the others, not showing the shoulder observed in the other two curves. The two-steps melting observed in the DSC curves suggest the presence of two different ordering of the crystallites. Probably one refers to the melting of the crystallites in the polymer bulk and the other to the transcrystallinity phase, respectively. Therefore, it is possible to conclude that the surface of the carbon fiber favored the crystallites nucleation when slowly cooled.

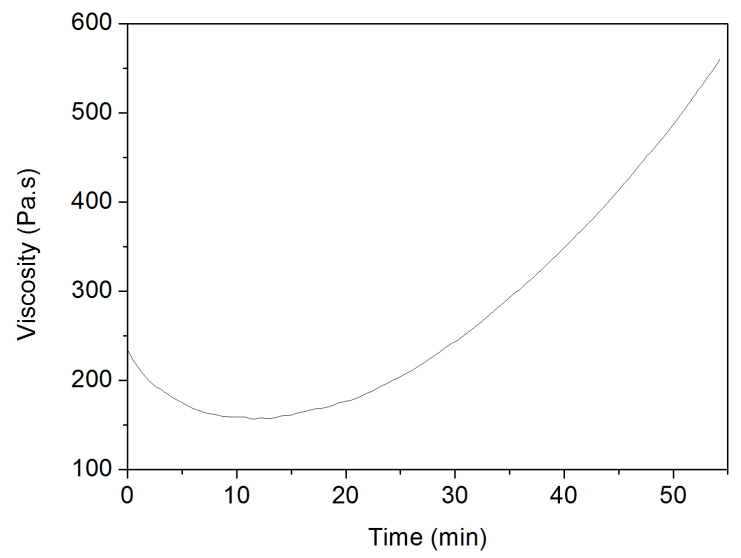

Figure 5. Viscosity behavior of melted PPS at $315^{\circ} \mathrm{C}$.

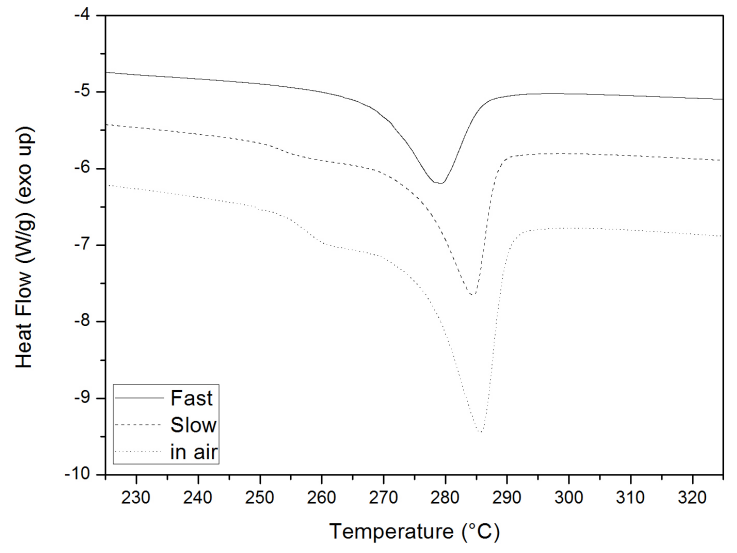

Figure 6. Effect of the cooling rate on the DSC curves of CF/PPS composites.

Table 1. Effect of the cooling rate on the crystallinity content of PPS matrix in the composite.

\begin{tabular}{cc}
\hline Cooling mode & $\mathbf{X}_{\mathbf{c}} \%$ \\
\hline Fast & $51.1 \pm 1.0$ \\
Slow & $58.5 \pm 0.8$ \\
In Air & $61.9 \pm 1.9$ \\
\hline
\end{tabular}

\subsection{Dynamic mechanical properties}

DMA tests were performed in order to evaluate the viscoelastic behavior of the composites as a function of temperature. Dynamic mechanical tests are sensitive to several transitions and relaxation processes of the polymer in the composite, and provide information about the fiber/ matrix interface ${ }^{18}$. Figure 7(a) shows the temperature dependence of the storage modulus (E'). The storage modulus is indicative of the elastic energy stored in the
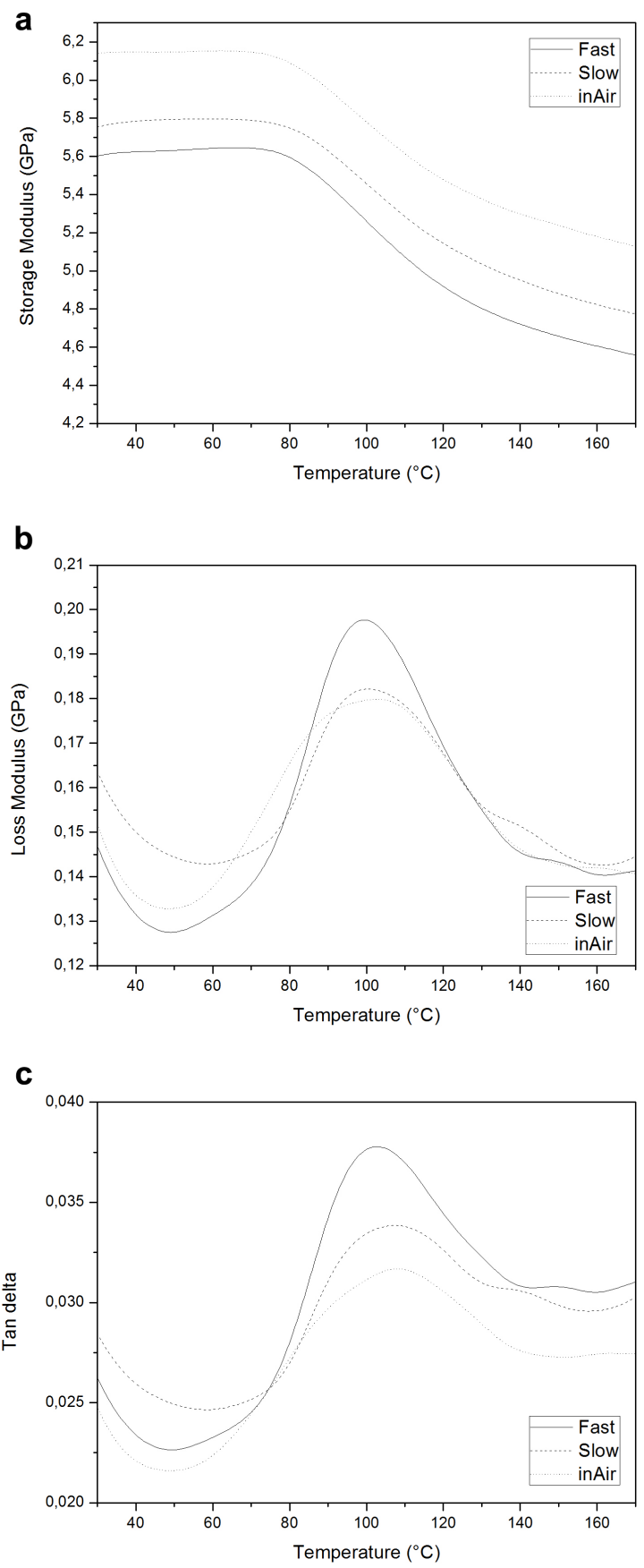

Figure 7. Effect of the cooling rate on the: (a) storage modulus; (b) loss modulus; and (c) $\tan \delta$ values of PPS/carbon fiber composites. 
material and it is affected by changes in the morphology of the material, induced, for example, by the crystalline content. Regarding the different laminates, significant E' increments are found for the samples with higher crystalline content. This result shows that slower cooling rates favored the stiffness increasing. Furthermore, it can be concluded that different cooling rates affected the crystallinity content of PPS matrix and consequently the mechanical behavior of the PPS/carbon fiber composite.

Figure 7(b) shows the variation of the loss modulus (E") with the temperature. The loss modulus is related to the energy dissipation mechanisms in the material. Therefore, composites with poor interfacial bonding tend to dissipate more energy. The experimental data indicates that the composite laminates with higher crystalline content (cooled slowly and in air, respectively) display lower loss modulus, suggesting that an improvement on the fiber/matrix interface occurred mainly as a result of PPS polymer crystallites nucleation on the carbon fibers.

The ratio between loss modulus and storage modulus is defined as $\tan \delta$ and it is an indicative parameter of the mechanical damping ability of the material. The damping properties provide the balance between the elastic and viscous phases in a polymeric structure. The evolution of $\tan \delta$ as a function of temperature is shown in Figure 7(c). In comparison with the fast cooled CF/PPS, the height of $\tan \delta$ peak decreases for both the slow cooled and in air cooled laminates, respectively. Therefore, the results reveal a decrease of the damping ability with the increase of the crystalline content. As the damping is an indicative of the amount of energy used to deform the material that is directly dissipated into heat, these results are found to be coherent.

\subsection{Young's modulus measurements}

The Young's modulus (E), measured by impulse excitation vibration, was also investigated. The results obtained are shown in the summary of the mechanical test results (Table 2). The Young's modulus of the laminates cooled slowly and in air experience a raise of about $5 \%$ and $9 \%$, respectively, when compared with the fast cooled. These increments are attributed to the higher stiffness promoted by the increase of the crystallinity content. This result is in agreement with the behavior observed for E', obtained by DMA. Thus, the increase of crystallinity corroborates to a higher modulus, storage and Young's, and, consequently, to an improvement on the mechanical resistance.

\subsection{Interlaminar shear strength}

To evaluate the fiber/matrix interface of the laminates, ILSS tests were performed and the quantitative results included in Table 2. The interlaminar shear strength of the

Table 2. Young's modulus and ILSS values of the CF/PPS composites obtained with different cooling rates.

\begin{tabular}{ccc}
\hline Cooling mode & E $(\mathbf{G P a})$ & ILSS $(\mathbf{M P a})$ \\
\hline Fast & $63.8 \pm 0.6$ & $27.5 \pm 2.1$ \\
Slow & $67.2 \pm 0.7$ & $28.1 \pm 3.3$ \\
In Air & $69.7 \pm 0.9$ & $31.4 \pm 1.9$ \\
\hline
\end{tabular}

CF/PPS laminates increased about $14 \%$ when the cooling mode changed from fast to in air. However, statistically speaking, no variation in strength was observed concerning the cooling change from fast to slow. Thus, the cooling in air probably favored the nucleation of crystallites on the surface of the carbon fibers and the crystallization formation along the polymer/fiber interface, also known as transcrystalline layer. Comparing the slow and in air cooling curves on Figure 1, it is possible to suppose that the transcrystallinity formation was not only due to the low cooling rate, since both profiles did not present a significant variation in the first $30 \mathrm{~min}$ of temperature fall. The absence of the cooling system utilization probably contributed to promote a stable environment, favoring the transcrystallinity formation. A similar behavior was observed by polarized light microscopy, as depicted in Figure 3, when the bubble on the surface of the fiber provided not just a thinner layer of polymer but also a more stable environment, favoring the crystallites nucleation and growth. As a result of the transcrystalline layer, the interface of the laminates was enhanced, leading to an improvement in the ILSS values. This result is in line with previous E" analysis, where an improvement in a mechanical property caused by the fiber/matrix interface enhancement was also inferred.

Overall, this study has shown the determinant effect of the cooing rate on the degree of crystallinity of PPS matrix in $\mathrm{CF} / \mathrm{PPS}$ laminates and, as a consequence, its fundamental role on the improvement of the mechanical and thermo-mechanical performances of the processed composite laminates.

\section{Conclusions}

The morphological aspects and the thermal and mechanical properties of CF/PPS laminates with different crystalline degrees have been investigated. Previous works have discussed about transcrystallization in $\mathrm{CF}$ / PPS composites and the degree of crystallinity influence on the mechanical properties ${ }^{21-24}$. In the present work, morphological observations revealed a highly homogeneous crystallization process for PPS, which posed some difficulties in the visualization of crystallites nucleation on the surface of the carbon fiber. Thus, the identification of nucleation and transcrystalline layer formation was only possible in a more stable environment with a considerably thinner polymer film. DSC results confirmed the increase of the crystallinity contents for the samples obtained with lower cooling rates and showed that the crystallization occurred in two steps for the specimens with higher crystalline degree (slower cooling rate). This result indicates the presence of different ordering of the crystallites, probably due to the presence of them in the polymer bulk and also the transcrystalline layer formation. DMA experiments showed an increase in the storage modulus and a decrease in both the loss modulus and $\tan \delta$ with the increment of the crystalline degree. This behavior was a result of the stiffness increase and the improvement of the fiber/matrix interface. The Young's modulus and the ILSS also increased mainly due to the improvements in stiffness and fiber/matrix interface, respectively. Overall, by changing only one parameter of 
the PPS/carbon fiber processing, the cooling rate, it was possible to induce a significant improvement in the laminate mechanical resistance. The mechanical tests demonstrated enhancements in stiffness, strength and fiber/matrix interface for the laminates processed with lower cooling rates.

\section{References}

1. Anagreh N, Dorn L and Bilke-Krause C. Low-pressure plasma pretreatment of polyphenylene sulfide (PPS) surfaces for adhesive bonding. International Journal of Adhesion and Adhesives. 2007; 28(1-2):16-22.

2. Díez-Pascual AM, Naffakh M, Marco C and Ellis G. Mechanical and electrical properties of carbon nanotube/poly(phenylene sulphide) composites incorporating polyetherimide and inorganic fullerene-like nanoparticles. Composites. Part A, Applied Science and Manufacturing. 2012; 43(4):603-612.

3. Díez-pascual AM and Naffakh M. Synthesis and characterization of nitrated and aminated poly(phenylene sulfide) derivatives for advanced applications. Materials Chemistry and Physics. 2012; 131(3):605-614.

4. Kim NH, Kuila T, Kim KM, Nahm SH and Lee JH. Material selection windows for hybrid carbons/poly(phenylene sulfide) composite for bipolar plates of fuel cell. Polymer Testing. 2012; 31(4):537-545.

5. Yilmaz T and Sinmazçelik T. Geometric parameters and chemical corrosion effects on bearing strength of polyphenylenesulphide (PPS) composites. Materials \& Design. 2007; 28:1695-1698.

6. Yang Y, Duan H, Zhang S, Niu P, Zhang G, Long S, et al. Morphology control of nanofillers in poly (phenylene sulfide): A novel method to realize the exfoliation of nanoclay by $\mathrm{SiO}_{2}$ via melt shear flow. Composites Science and Technology. 2013; 75:28-34.

7. Díez-pascual AM and Naffakh M. Tuning the properties of carbon fiber-reinforced poly(phenylene sulphide) laminates via incorporation of inorganic nanoparticles. Polymer. 2012; 53(12):2369-2378.

8. Vieille B, Aucher J and Taleb L. Influence of temperature on the behavior of carbon fiber fabrics reinforced PPS laminates. Materials Science and Engineering A. 2009; 517(1-2):51-60.

9. Vieille B, Albouy W, Chevalier L and Taleb L. About the influence of stamping on thermoplastic-based composites for aeronautical applications. Composites. Part B, Engineering. 2013; 45(1):821-834.

10. Van Dommelen JA, Parks DM, Boyce MC, Brekelmans WA and Baaijens FP. Micromechanical modeling of intraspherulitic deformation of semicrystalline polymers. Polymer. 2003; 44(19):6089-6101.

11. Li L, Chan CM, Yeung KL, Li JX, Ng KM and Lei Y. Direct observation of growth of lamellae and spherulites of a semicrystalline polymer by AFM. Macromolecules. 2001; 34(2):316-325.

\section{Acknowledgements}

The authors would like to thank CNPq (Process numbers 303287/2013-6 and 303559/2012-8), FAPESP (Process number 2012/12207-6) and CAPES (Process number 12357-13-8) for the financial support.

12. Ma UVL, Floros JD and Ziegler GR. Effect of starch fractions on spherulite formation and microstructure. Carbohydrate Polymers. 2011; 83(4):1757-1765.

13. Ning N, Fu S, Zhang W, Chen F, Wang K, Deng H, et al. Realizing the enhancement of interfacial interaction in semicrystalline polymer/filler composites via interfacial crystallization. Progress in Polymer Science. 2012; 37(10):1425-1455.

14. Gao SL and Kim JK. Cooling rate influences in carbon fibre/ PEEK composites. Part 1. Crystallinity and interface adhesion. Composites. Part A, Applied Science and Manufacturing. 2000; 31(6):517-530.

15. Quan H, Li ZM, Yang MB and Huang R. On transcrystallinity in semi-crystalline polymer composites. Composites Science and Technology. 2005; 65(7-8):999-1021.

16. Fujihara K, Huang ZM, Ramakrishna S and Hamada H. Influence of processing conditions on bending property of continuous carbon fiber reinforced PEEK composites. Composites Science and Technology. 2004; 64(16):2525-2534.

17. Dai P, Zhang W, Pan Y, Chen J, Wang Y and Yao D. Processing of single polymer composites with undercooled polymer melt. Composites. Part B, Engineering. 2011; 42(5):1144-1150.

18. Díez-Pascual AM, Ashraft B, Naffakh M, González-Domíngez JM, Johnston A, Simard B, et al. Influence of carbon nanotubes on the thermal, electrical and mechanical properties of poly(ether ether ketone)/glass fiber laminates. Carbon. 2011; 49(8):28172833.

19. Hu T, Juuti J and Jantunen H. RF properties of BST-PPS composites. Journal of the European Ceramic Society. 2007; 27(8-9):2923-2926.

20. Stoeffler K, Andjelic S, Legros N, Roberge J and Schougaard SB. Polyphenylene sulfide (PPS) composites reinforced with recycled carbon fiber. Composites Science and Technology. 2013; 84:65-71.

21. Schulz E, Kalinka G and Auersch W. Effect of transcrystallization in carbon fiber reinforced poly(p-phenylene sulfide) composites on the interfacial shear strength investigation with the single fiber pull-out test. Journal of Macromolecular Science, Part B: Physics. 1996; 35:527-546.

22. Boey FY, Lee TH and Yue CY. Effect of matrix crystallinity on the buckling failure of a PPS thermoplastic composite. Polymer Testing. 1994; 13(1):47-53.

23. Vieille B, Aucher J and Taleb L. Influence of temperature on the behavior of carbon fiber fabrics reinforced PPS laminates. Materials Science and Engineering A. 2009; 517(1):51-60.

24. Boey FYC, Lee TH and Yue CY. Annealing effects on the dynamic mechanical properties of aromatic polyphenylene sulphide fibre reinforced composite. Polymer Testing. 1991; 10(19):221-228. 